\title{
EFICIÊNCIA DE ÉSTERES DE SACAROSE EM AMEIXAS (Prunus salicina, Lindl.) 'SANTA ROSA' REFRIGERADAS
}

\author{
R.A. KLUGE ${ }^{1}$; R.F.F. CANTILLANO ${ }^{2}$; R.O. JORGE ${ }^{3}$ \\ 'Depto. de Horticultura, ESALQUUS, C.P. 9, CEP: 13418-900, Piracicaba, SP \\ ${ }^{2}$ EMBRAPA/CPACT, C.P. 403, CEP 96001-970, Pelotas, RS \\ ${ }^{3}$ FAEM/UFPEL, C.P. 354, CEP: 96001-970, Pelotas, RS
}

RESUMO - Com o objetivo de avaliar o efeito do TAL-PROLONG (ésteres de sacarose), ameixas 'Santa Rosa' foram armazenadas por 21 dias a $0^{\circ} \mathrm{C}$ e 90-95\% UR (+ 3 dias de comercialização simulada em temperatura ambiente). As doses do TALPROLONG utilizadas foram $0 ; 0,5 ; 1,0 ; 1,5$ e 2,0\%, aplicadas antes do armazenamento por imersāo das frutas. As frutas tratadas com TAL-PROLONG apresentaram menores perdas de peso durante 0 armazenamento e comencializaçăo simulada. Houve menor incidência de podridōes e desintegraçăo interna de polpa nas frutas tratadas com TAL-PROLONG, o qual foi também eficiente na manutenção da coloração das frutas.

Descritores - Esteres de sacarose, desintegração interna, perda de peso, ameixas, Prunus salicina, armazenamento.

\section{EFFICIENCY OF SUCROSE ESTERS IN PLUMS (Prunus salicina, Lindl.) 'SANTA ROSA' COLD STORED}

ABSTRACT - Aiming to verify the efficiency of TAL-PROLONG (sucrose esters), plums 'Santa Rosa' were stored for 21 days at $0^{\circ} \mathrm{C}$ and $90-95 \%$ RH ( 3 days of simulated marketing at room temperature). The fruits were immersed in a solution containing $0 ; 0,5 ; 1,0 ; 1,5$ and $2,0 \%$ of TAL-PROLONG, before storage. During cold storage and marketing, fruits treated with TAL-PROLONG presented less weight loss. The decay and internal breakdown incidence was reduced in fruits treated with TAL-PROLONG and it was efficient for maintening the fruit colour.

Key words - sucrose esters, internal breakdown, weight loss, plums, Prunus salicina, storaging.

\section{INTRODUÇĀO}

Ameixas, e outras frutas de caroço, geralmente possuem baixa capacidade de armazenamento devido a problemas de desidratação, distúrbios fisiologicos e podridões que aparecem em pós-colheita (HARDENBURG et al., 1986; CANTILLANO, 1987).

A perda de peso fresco, decorrente dos processos transpiratório e respiratório pode, em alguns casos, levar ao murchamento e perda de consistência da fruta, diminuindo a sua aceitabilidade comercial (WOODS, 1990; AWAD, 1993).

O distúrbio fisiológico mais importante em ameixas é a desintegração interna de polpa ("Internal Breakdown"). Esta é caracterizada pela desorganização celular, decorrente da perda da semi-permeabilidade das membranas e da rigidez das paredes celulares, tornando escurecidos e impalatáveis os tecidos da polpa da fruta (EKSTENN, 1982; DODD, 1984). A susceptibilidade varietal à desintegração interna é um dos determinantes do potencial de conservação de ameixas (GATTI \& ESCUDERO, 1985). Sabese atualmente que este distúrbio é causado pelas próprias condições de temperatura de armazenamento. Para o armazenamento de frutas de clima temperado em geral, são utilizadas baixas temperaturas, próximas a $0^{\circ} \mathrm{C}$, e estas podem causar danos denominados injúrias pelo frio ou "chilling", resultando numa série de disfunções, dentre elas o aparecimento da desintegração interna de polpa (GATTI \& ESCUDERO, 1985; WANG, 1991; LUZA et al., 1992; CRISOSTO, 1994). A temperatura baixa reduz demasiadamente muitas atividades enzimáticas importantes, causando modificações nas membranas e favorecendo a acumulação de intermediários tóxico que danificam os tecidos das frutas (CHITARRA \& CHITARRA, 1990; GRAHAM, 1990; WANG, 1991). Não só a temperatura de armazenamento determina os sintomas do "chilling", mas também o tempo de exposição das frutas à frigoconservação (CHITARRA \& CHITARRA, 1990).

$A$ presença de fungos durante 0 armazenamento refrigerado, principalmente os dos gêneros Monilinia, Rhizopus, Botrytis e Penicillium 
$\epsilon$ comum em ameixas armazenadas e contribui sobremaneira para a perda de qualidade das frutas (TUSET, 1992).

Como forma de diminuir os problemas acima citados com vistas ao prolongamento do período de armazenamento e comercialização de ameixas está, entre outros métodos, a utilização da atmosfera modificada. A atmosfera modificada 6 uma prática complementar à refrigeração e tem como objetivo principal retardar os ritmos metabólicos da maturação e senescência das frutas através da modificação da atmosfera que a rodeia, com elevação nos níveis de $\mathrm{CO}$ e/ou $\mathrm{O}$. Com isso é reduzido a taxa respiratória e a próduçâo de etileno pelas frutas (DINAMARCA et al., 1989). Para a obtenção de atmosfera modificada pode-se utilizar materiais plásticos, cêras ou películas protetoras. Dentre as películas protetoras destaca-se aquelas a base de ésteres de sacarose, derivados de ácidos graxos, carboximetilcelulose sódica e mono e diglicerídeos. No mercado internacional pode-se encontrar produtos a base de ésteres de sacarose sob o nome técnico de TAL-PROLONG ou Semperfresh. Estes produtos tem apresentado resultados promissores em diversas frutas, mantendo a sua consistência, reduzindo a incidência de fungos e distúrbios fisiológicos e evitando perdas elevadas de peso fresco (BANKS, 1984, 1985; SMITH et al., 1987; MEHERIUK \& LAU, 1988; DINAMARCA et al., 1989; KRISHNAMURTHY, 1989). DINAMARCA et al. (1989) verificaram efeito benéfico da aplicação de ésteres de sacarose na manutenção da firmeza e cor da epiderme para ameixas 'Friar', 'Laroda', 'Larry Ann', 'Royal Diamond' e 'Angelino' enquanto que para 'Nubiana' não houve efeito do produto. Segundo os mesmos autores, o efeito do produto depende do cultivar, devendo ser testado em condições locais.

O objetivo deste trabalho foi verificar a influência da aplicação de ésteres de sacarose sobre alguns aspectos de qualidade durante a frigoconservação de ameixas 'Santa Rosa'.

\section{MATERIAL E MÉTODOS}

O trabalho foi realizado nas câmaras frigoríficas da CESA - Companhia Estadual de Silos e Armazém e nos laboratórios da EMBRAPA/ CPACT, em Pelotas, RS. Ameixas 'Santa Rosa', oriundas da safra 1992/93, foram colhidas no estádio verde-maturo, com $50-75 \%$ de coloração vermelha, tratadas com Benlate $(0,6 \%)+$ Allisan $(0,13 \%)$ e armazenadas por um período de 21 dias a $0^{\circ} \mathrm{C}$ e $\mathbf{9 0 - 9 5 \%}$ UR. Após a retirada do armazenamento, os frutos foram submetidos a um período de comercialização simulada de 3 dias, sob temperatura ambiente $\left(24-25^{\circ} \mathrm{C}\right.$ e $60-70 \%$ UR).

Os frutos foram colocados em redes plásticas, na proporção de 15 frutos por rede, correspondendo à repetição. Nos tratamentos, foi utilizado o TAL-PROLONG, a base de ésteres de sacarose, aplicado na forma de solução, em imersão dos frutos por 30 segundos, nas dosagens de 0 (água destilada); 0,$5 ; 1,0 ; 1,5$ e $2,0 \%$, sendo logo após submetidos a uma secagem com ar forçado durante 5 minutos.

Os parâmetros avaliados ao final do armazenamento foram:

a) cor da epiderme: avaliou-se o percentual de coloração vermelha cobrindo as frutas, utilizadose uma escala subjetiva de 1 a 3 , correspondendo a 50,70 e $100 \%$ de coloração vermelha; e intensidade de coloração por comparação com Tabelas de cores do Código Universal de Cores (SEGUY, s.d.), com escala compreendendo os valores entre 31 (vermelho pouco intenso) até $\mathbf{4 2}$ (vermelho muito intenso);

b) Perda de peso: expressa em porcentagem, segundo as fórmulas:

$\mathrm{PPA}=[(\mathrm{PI}-\mathrm{PS}) / \mathrm{PI}] \times 100$ e $\quad \mathrm{PPCS}=[(\mathrm{PS}-$ PCS)/PS $] \times 100$

onde:

PPA = Perda de peso durante o armazenamento; PI = Peso inicial da repetição;

PS = Peso da repetição imediatamente na saída do armazenamento;

PPCS $=$ Perda de peso durante a comencialização simulada;

PCS = Peso da repetição após 3 dias de comercialização simulada.

c) podridões: foram consideradas a fetadas aquelas com sintomas visíveis e expressa em porcentagem; d) desintegração interna de polpa: após o corte das frutas e expressa em porcentagem.

Forma utilizados 5 tratamentos com 4 repetições de 15 frutas cada. Os dados coletados foram submetidos à análise de variação. Para 0 estudo do efeito de doses de TAL-PROLONG foi utilizada a regressão polinomial. 


\section{RESULTADOS E DISCUSSÃO}

Cor: As frutas que não foram tratadas com esteres de sacarose apresentaram-se completa e intensamente vermelhas ao final dos 21 dias de armazenamento refrigerado e comercialização. Nas frutas tratadas, houve uma manutenção da coloração, principalmente quando se usou as doses 1,$0 ; 1,5$ e 2,0\% (TABELA 1). Estes resultados concordam com os relatados por DINAMARCA et al. (1989), que observaram retardo do desenvolvimento de coloração para alguns cultivares de ameixas quando da utilização de ésteres de sacarose durante o armazenamento das frutas.

TABELA 1. Efeito de ésteres de sacarose sobre a coloração em ameixas 'Santa Rosa' armazenadas por 21 dias a $0^{\circ} \mathrm{C}$ e $90-95 \%$ UR.

\begin{tabular}{lccccc}
\hline \hline \multirow{2}{*}{$\begin{array}{c}\text { Coloração } \\
\text { da epiderme }\end{array}$} & 0 & 0,5 & 1,0 & 1,5 & 2,0 \\
\cline { 2 - 6 } & 100 & 75 & $50-75$ & $50-75$ & $50-75$ \\
\hline $\begin{array}{l}\text { \% de coloração } \\
\text { vermelho }\end{array}$ & 42 & 37 & 36 & 31 & 31 \\
\begin{tabular}{l} 
Intensidade \\
\cline { 2 - 6 }
\end{tabular} & 42 & \multicolumn{5}{c}{ Dose de ésteres de sacarose (\%) } \\
\hline \hline
\end{tabular}

142 = mais intenso e 31 = menos intenso, segundo SÉGUY (s.d.)

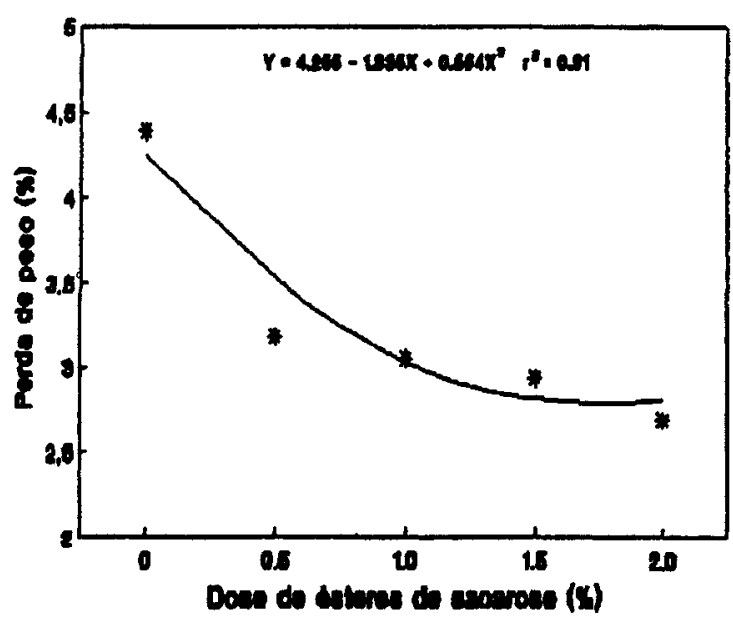

Figura 1 - Efeito de ésteres da sacarose sobre a perda de peso em ameixas 'Santa Rosa' durante o armazenamento por 21 dias a $0^{\circ} \mathrm{C}$ e $90-95 \%$ UR.

Perda de peso: A perda de peso das frutas foi reduzida quando utilizou-se ésteres da sacarose em todas as dosagens, tanto durante $o$ armazenamento quanto na comercialização simulada (FIGURAS 1 e 2). Segundo BANKS (1984), os produtos a base de ésteres de sacarose formam uma película que bloqueia os estômatos impedindo a desidratação das frutas. Houve efeito residual do produto nos três dias de comercialização, com menores perdas de peso a medida que aumentou-se a dose de ésteres de sacarose (FIGURA 2). Os resultados do presente experimento, para este parâmetro, contrariam DINAMARCA et al. (1989), que referiram aos produtos à base de ésteres de sacarose como não redutores da desidratação de ameixas.

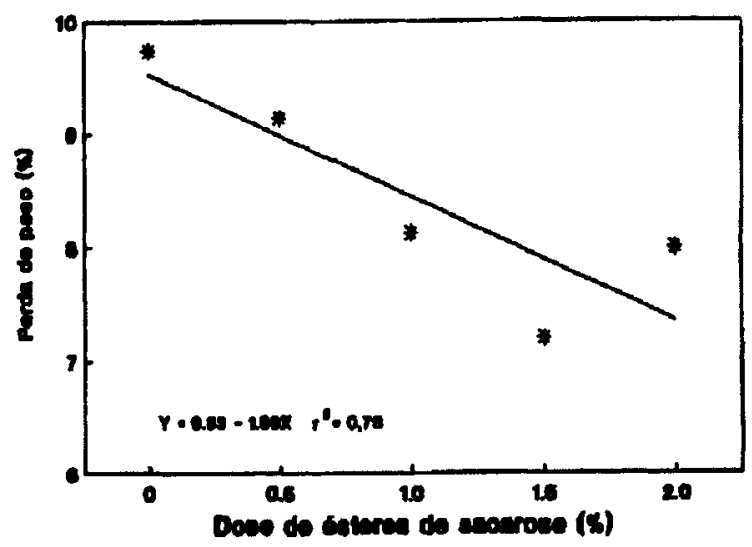

Figura 2 - Efeito de ésteres de sacarose sobre a perda de peso de ameixas 'Santa Rosa' durante a comercialização simulada por 3 dias em temperatura ambiente (24$\left.25^{\circ} \mathrm{C}\right)$ e $60-70 \%$ UR, após armazenamento refrigerado por 21 dias. 
Podridōes: $O$ aumento na dose de ésteres de sacarose reduziu a incidência de podridões, alcançando valores inferiores a $5 \%$ (FIGURA 3 ). Segundo SOMMER (1982), a diminuição de $\mathrm{O}_{2}$ e/ ou a elevação de $\mathrm{CO}_{2}$ reduzem o crescimento e/ou germinação de esporos de fungos. Provavelmente, as doses maiores da película protetora utilizada no experimento promoveu um acúmulo de $\mathrm{CO}_{2}$ suficiente para causar um efeito fungistático sobre os fungos existentes. Os fungos mais frequentes encontrados durante o armazenamento foram Penicillium sp, Alternaria sp e Monilinia sp.

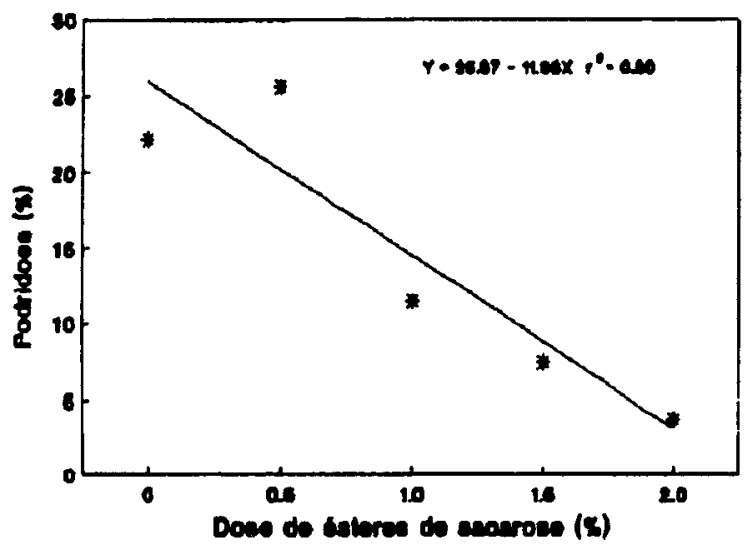

Figura 3 - Efeito de ésteres de sacarose sobre a incidência de podridões em ameixas 'Santa Rosa' armazenadas por 21 dias a $0^{\circ} \mathrm{C}$ e 3 dias de comercialização simulada em temperatura ambiente.

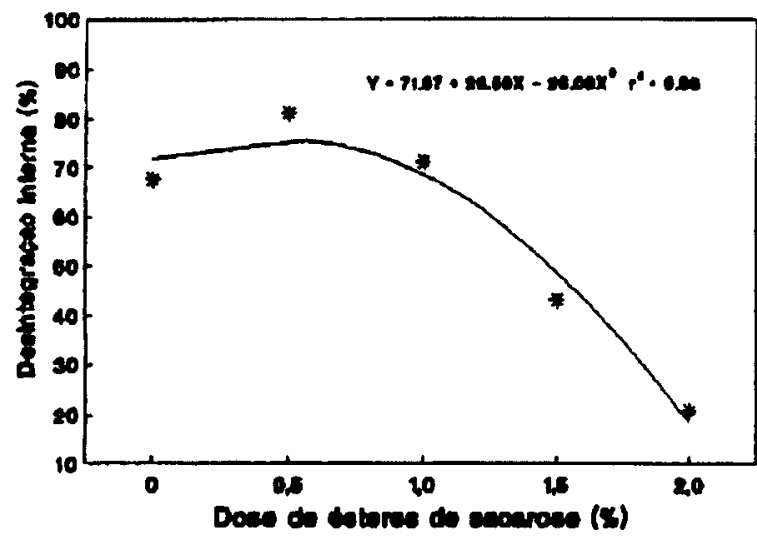

Figura 4 - Efeito de ésteres de sacarose sobre a incidência de desintegração interna em ameixas 'Santa Rosa' armazenadas por 21 dias a $0^{\circ} \mathrm{C}$ e 3 dias de comercialização simulada em temperatura ambiente.
Desintegração interna: $O$ aumento nas doses de ésteres de sacarose também diminuiu a percentagem de frutos com sintomas de desintegração interna, atingindo na dose de $2 \%$, valores próximos a $20 \%$ (FIGURA 4). É provável que a modificação das condições atmosféricas $\left(\mathrm{O}_{2} / \mathrm{CO}_{2}\right)$, originadas como o uso da película protetora, tenha exercido algum controle sobre esse distúrbio fisiológico através da redução do ritmo metabólico das frutas, principalmente os de síntese de compostos que poderiam danificar os tecidos internos.

\section{CONCLUSÕES}

A aplicação de ésteres de sacarose antes do armazenamento refrigerado de ameixas 'Santa Rosa' reduz a incidência de podridões causadas por fungos, a desintegração interna de polpa e as perdas de peso das frutas, além de manter a sua coloração.

\section{REFERÊNCIAS BIBLIOGRÁFICAS}

AWAD, M. Fisiologia pós-colheita de frutos. São Paulo: Nobel, 1993. 114p.

BANKS, N.H. Studies of the banana fruit surface in relation to the effects of Tal-Prolong coating on gaseous exchange. Scientia Horticulturae, v.24, n.3/4, p. 279 $86,1984$.

BANKS, N.H. Internal atmosphere modification in prolong coated apples. Acta Horticulturae, n. 517, p. $105-12,1985$.

CANTILLANO, R.F. Fisiologia e manejo de pós-colheita de ameixas. Pelotas, EMBRAPA,CNPFT, 1987. 10p.

CHITARRA, M.I.F.; CHITARRA, A.B. Pós-colheita de frutos e hortaliças: fisiologia e manuseio. Lavras: ESAL,FAEPE, 1990. 320p.

CRISOSTO, C.H. Factores que afectan la calidad de la fruta y su deterioro en postcosecha. In: CURSO INTERNACIONAL DE FRUTALES DE CAROZO, General Roca, 1994. Anais. General Roca: s.ed., 1994. cap. 7.1, p.1-10.

DINAMARCA, E.A.; MTTCHELL, F.G.; KADER, A.A. Use of sucrose esters as delaying agents of ripening of pears and plums. Revista Fruticola, v.10, n.3, p. 116-21, 1989.

DODD, M.C. Internal breakdown in plums. The Deciduous Fruit Grower, v.34, n.8, p.255-6, 1984. 
EKSTENN, G.J. Interna! breakdown of plums. The Deciduous Fruit Grower, v.32, n.9, p.359-61, 1982.

GATTI, R.; ESCUDERO, P. Pardeamiento interno en frutales de carozo. Revista Fruticola, v.6, n.2, p.458, 1985.

GRAHAM, D. Chilling injury in plantas and fruits: some possible causes with means of amelioration by manipulation of postharvest storage conditions. In: INTERNATIONAL CONGRESS OF PLANT PHYSIOLOGY, New Delhi, 1990. Proceedings. New Delhi:editora, 1990. v. 2, p.1373-84.

HARDENBURG, R.E.; WATADA, A.E.; WANG, C.Y. The commercial storage of fruits, vegetables, and florist, and nursery stocks. Washington:USDA, 1986. $130 \mathrm{p}$.

KRISHNAMURTHY, S. Effects of Tal-prolong on shelflife and quality arributes of mango. Acta Horticulturae, n 231, p. 675.8, 1989.

LUZA, J.G.; GORSEL, R. von, POLITO, V.S.; KADER, A.A. Chilling injury in peaches: A cytochemical and ultrastructural cell wall study. Jourmal of the American Society for Horticultural Science, v.117, n.1, p.114-8, 1992.

MAKHART, A.H. Chilling injury: a review of possible causes. HortScience, v.26, n.6, p.1329-33, 1986.

MEHERIUK, M.; LAU, O.L. Effect of two polymeric coating on fruit quality of 'Bartlett' and 'd'Anjou' pears. Journal of the American Society for Horticultural Science. v.113, n. 2, p. 222-6, 1988.
SÉGUY, E. XXX Code Universel des couleurs. Paris: Paul Lechevalier, s.d. 48 p.

SMTH, S.; GEESON, J.; STOW, J. Production of modified atmosphere in deciduous fruit by the use of films and coating. HortScience, v. 22, n.5, p.772-6, 1987.

SOMMER, N.F. Postharvest handling practices and postharvest deseases of fruits. Plant Disease, v.66, n.5, p.357-64, 1982.

TUSET, J.J. Estrategia de control de las principales podredumbres de frutas y hortalizas. In: CURSO DE PATOlOgIA DE POSCOSECHA DE HORTALIZAS Y FRUTAS DE CLIMA TEMPLADO, 1. , Montevideo, Anais. Montevideo: SUH' COLHOR, 1992. p.6-24.

WANG, C.Y. Reduction of chilling injury in fruits and vegetables. Postharvest News and Information, v.2, n.3, p.165-8, 1991.

WOODS, J.L. Moisture loss from fruits and vegetables. Postharvest News and Information, Wallingford, v.1, n.3, p.195-9, 1990.

Recebido para publicação em 26.07.95 Aceito para publicaçào em 14.08 .95 